\title{
Aberrant death in dark chondrocytes of the avian growth plate
}

\author{
Jekaterina Erenpreisa ${ }^{1}$ and Helmtrud I. Roach ${ }^{2}$ \\ 1 A. Kirchenstein Institute of Microbiology and Virology, Riga, LV-1067, Latvia \\ 2 Academic Orthopaedic Unit, CF86, Southampton General Hospital, S016 6YD, \\ UK \\ ${ }^{3}$ corresponding author: Dr Jekaterina Erenpreisa, A. Kirchenstein Institute, \\ Ratsupites 1, Riga, LV-1067; fax: (3712) 428036; \\ e-mail: cancer@laima.acad.latnet.Iv
}

Received 13.4.97; revised 28.8.97; accepted 8.9.97

Edited by R.A.Knight

\begin{abstract}
Growth plate chondrocytes of embryonic chick femurs were examined by electron microscopy, cytophotometry and autoradiography. Apart from the well-described 'light' chondrocyte, a different 'dark' type of chondrocyte was present, comprising $10-35 \%$ of the cell population. They were found at all stages of chondrocyte differentiation and in all ages of the femurs studied. Well developed rough endoplasmatic reticulum and Golgi complex, many secretory vesicles, energetically active mitochondria and a lot of glycogen, indicating high activity of the cytoplasm, were combined with Iow RNA synthesis, gentle margination and scattered compaction of the chromatin. DNA cytometry revealed that most of dark cells were diploid, but $15-30 \%$ were tetraploid, with the absence of an S-phase. Substantial loss of DNA was found in about $10 \%$ of dark chondrocytes. The TUNEL reaction demonstrated a limited number of DNA strand breaks. Advanced dark cells possessed the nuclear features of both apoptosis and necrosis. Besides chromomeric-chromonemic compaction, a chromatin arrangement similar to that of prometaphase and metaphase, as well as amitotic nuclear segregation, all of them degenerative, were found. Our interpretation is that the dark chondrocytes undergo an aberrant type of cell death which may be combined with aberrant cell cycle. Cell death of dark chondrocytes is preceded by a pre-mortal burst of secretion.
\end{abstract}

Keywords: apoptosis; growth plate; chondrocyte; chick embryo; aberrant cell cycle

Abbreviations: TUNEL, Terminal transferase-mediated dUTP nick end labelling; RER, rough endoplasmatic reticulum; TEM, transmission electron microscopy

\section{Introduction}

Cells, which appear unusually dark in the electron microscope and in Toluidine blue-stained semi-thin sections, have been known since the last century as a characteristic feature of many tumours and healthy tissues (e.g. skin, liver, brain). A darkly stained cytoplasm and denser, smaller and at times also crenated nuclei were the main cytological features described in these cells. Dark cells were sometimes considered to be a fixation artefact (Ganote and Moses, 1968) or to represent a variant of the same cell type with otherwise normal morphology (Ghadially, 1988). Dark cells may comprise from 10 to $60 \%$ of tissue cells, increasing in number under functional or toxic load (Parsons et al, 1983). Johannisson (1968) suggested that the most advanced dark cells of the adrenal cortex containing swollen mitochondria and endoplasmic reticulum were almost certainly unviable. Other authors considered the dark cells to be functionally active, transiently damaged or stressed, apoptotic or necrotic (reviewed by Cooper et al, 1975; Parsons et al, 1983; Ghadially, 1988; Chentsov and Kosyh, 1995). In some tissues the appearance of dark cells was related to terminal differentiation, ageing or cancerogenesis (reviewed by Glaso and Hovig, 1987). In mouse mastocytoma, the formation of dark cells was thought to represent a third morphological pattern of cell death, in addition to necrosis and apoptosis (Harmon, 1987).

The fate of growth plate chondrocytes has engaged many researchers. It is still a point of controversy whether all chondrocytes undergo apoptosis (Farnum and Wilson, 1990; Gibson et al, 1995; Hatori et al, 1995; Bronkers et al, 1996; Aizawa et al, 1997) or whether some survive to become bone-forming cells (Cancedda et al, 1995; Roach et al, 1995). Using morphological criteria, any chondrocyte with a condensed nucleus is often considered unviable or apoptotic. However, with the exception of the erosion front, no convincing EM pictures of apoptotic chondrocytes in vivo have been presented hitherto.

The present study investigated the dark chondrocytes of the avian growth plate and addressed the question of whether the dark chondrocytes were a fixation artifact, represented a terminal stage of differentiation or a functional state and whether this state had any relation to cell death.

\section{Results}

\section{Influence of fixation}

The nuclear and cytoplasmic features of dark cells, described below, were preserved at any EM fixation modification including that with $\mathrm{RHT}$. In fact, using any method, the dark cells appeared more shrunk into the centre of their lacunae compared with the normal light chondrocytes. Reliably good EM results, diminishing shrinkage of cells were obtained by the prolonged (overnight) glutaraldehyde fixation with $A B+C A C l_{2}$. In the places of better fixation, some dark chondrocytes occupied the same volume as the neighbour- 

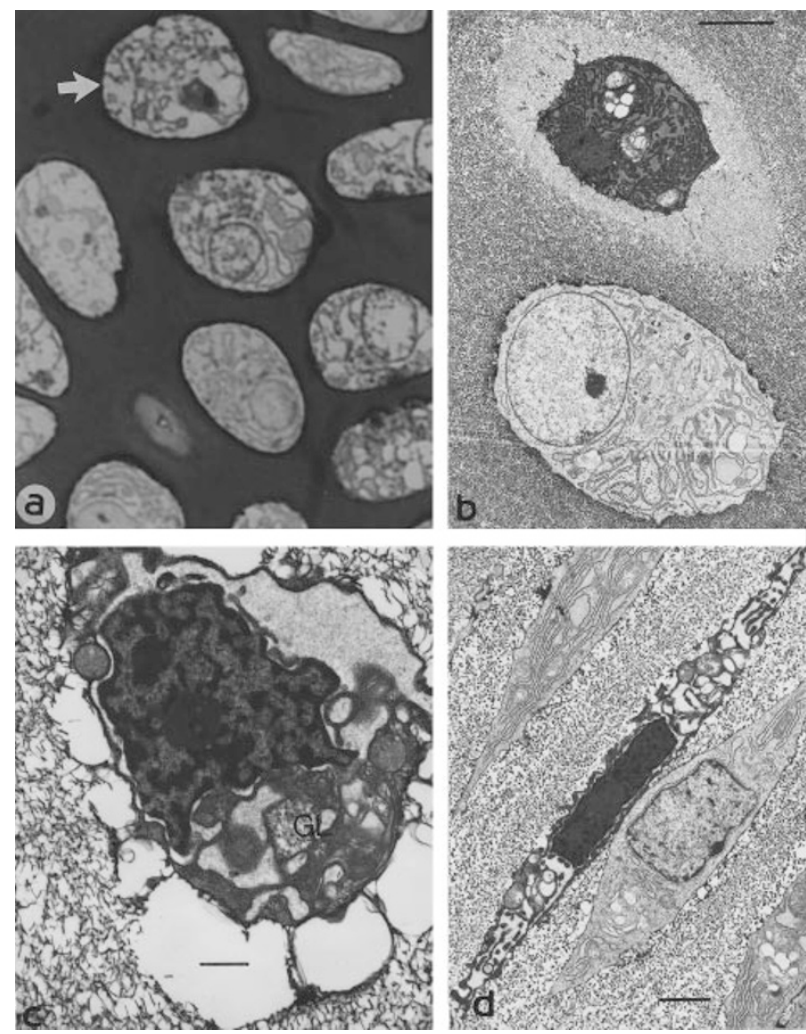

Figure 1 (a) A semithin-section of the hypertrophic region of the growth plate from a 14-d. chick embryo, fixed by $\mathrm{GA} / \mathrm{AB}+\mathrm{CaCl}_{2}$. A dark cell (arrow), recognized by typical pattern of nucleus and cytoplasm, is present in unshrunk form, magnification $\times 2500$. (b) Light and dark chondrocyte in the upper hypertrophy zone (14-d.embryo). In the dark cell, autophagosomes and swollen mitochondria are present. Bar: $5 \mu \mathrm{m}$. (c) A dark chondrocyte in a resting zone of 17-d. embryo. Gl-glycogen. Bar: $0.6 \mu \mathrm{m}$. (d) Light and dark chondrocytes in close proximity in proliferating zone (19-d. embryo). Bar: $1.5 \mu \mathrm{m}$

ing light hypertrophic chondrocytes, retaining, however, their characteristic morphology (Figure 1a). When varying the fixatives for cell imprints, the cytoplasmic basophilia of dark cells was easily extractable by organic solvents (ethanol/ acetone, less by methanol) and/or weak acid, suggesting the impairment of cell membranes and ribosomal attachment to them (data not shown).

\section{Distribution of dark chondrocytes in a growth plate}

Dark chondrocytes were present in the epiphyseal plates of chick embryos at all the ages studied. In 14 and 15-d chick embryos they comprised from 10 to $35 \%$ of the chondrocytes (counted in five animals). Dark chondrocytes were found adjacent to light ones in all zones of the growth plate, i.e. among resting, proliferating, maturing, and hypertrophic cells (Figures $1-2 b, 6)$. These were more numerous among proliferating cells. However, even in mineralisation areas, we could also distinguish both types of hypertrophic chondrocytes, light and dark, both possessing some signs of degeneration (Figure 2a, b). Dark cells were both scattered and clustered among light chondrocytes. Occasionally, one dark cell was found in a doublet with a light cell within one
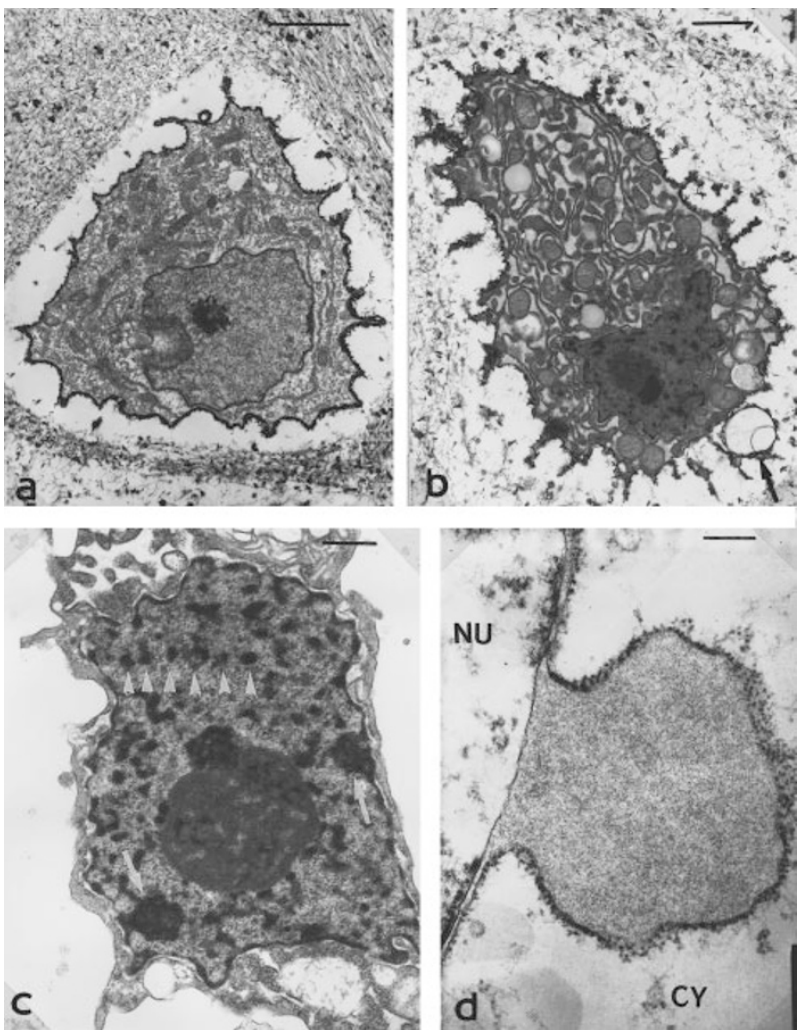

Figure 2 (a,b) Light and dark hypertrophic chondrocytes found in close proximity in the mineralisation zone (17-d. embryo): (a) 'light' chondrocyte possesses signs of initial degeneration displayed as detachment from lacuna walls, undulating and whorling nuclear envelope, dull, however evenly distributed chromatin and dissipation of a nucleolus; (b) 'dark chondrocyte' has different signs of degeneration, such as segregation of a nucleolus 'cap', single blebs of plasmalemma (arrow) and appears more viable than its 'light' counterpart. Bars: $0.7 \mu \mathrm{m}$. (c) A typical nucleus of dark cell: the tandemic arrangement of the chromatin condensations (arrowheads), the 'compact-type' large nucleolus, accessorial undeveloped nucleoli (arrows). Proliferating zone, 15-d. embryo. Bar: $0.7 \mu \mathrm{m}$. (d) Large bleb of the outer nuclear membrane of the light hypertrophic chondrocyte in the bottom region forming a secretory active cisterna of RER. NU - nucleus; CY-cytoplasm. 15-d. embryo. Bar: $0.2 \mu \mathrm{m}$

lacuna (data not shown). The morphology of the cartilage matrix around dark and light chondrocytes was undistinguishable by TEM.

By cell shape and size range, the dark cells were similar to, although usually smaller than, the light chondrocytes of the relevant layer. They were round in the resting zone, fusiform and flat in the proliferating zone, and enlarged and oval in the hypertrophic zone.

\section{Cytology of dark chondrocytes}

Nuclei The light chondrocytes had evenly shaped nuclei with highly dispersed chromatin (Figure 1b), sometimes with a few clumps. The dark chondrocytes, whether present near the perichondrium or in the late hypertrophic region, had a different but quite characteristic type of nuclear structure. They possessed an indented nuclear envelope, lined by gently marginated chromatin, and contained numerous chromomere-sized, $0.1 \mu \mathrm{m}$ in diameter, chromatin condensa- 
Dark chondrocytes $\mathrm{J}$ Erenpreisa and HI Roach tions within dense granular karyoplasm. A tandemic arrangement of the condensations was apparent in some sections (arrows in Figure 2c). The tandemic, chromomeric pattern of chromatin condensations has been previously documented by TV image analysis of the DNA-stained dark (named 'ageing') chondrocytes in 7-8 chick embryo femur growth plates (Erenpreisa and Zhukotsky, 1993). In more advanced dark cells, the density of karyoplasm increased and separate chromomeric patches tended to condense into the chromonema-like strands (Figure 1c) or, more seldom, into irregular clumps.

Nucleoli One or two centrally located nucleoli of highly varying types were present in light cells, but the same type of nucleolus was present in dark cells. Their nucleoli were large and composed mostly of the granular component (Figure 2c) representing the so-called 'compact nucleolus' - a type active in the maturing of pre-ribosomal particles (Hadjiolov, 1985). However, in later dark cells, nucleoli were smaller, segregated and/or hypercompacted signalling degeneration (Figure $2 b$ ).
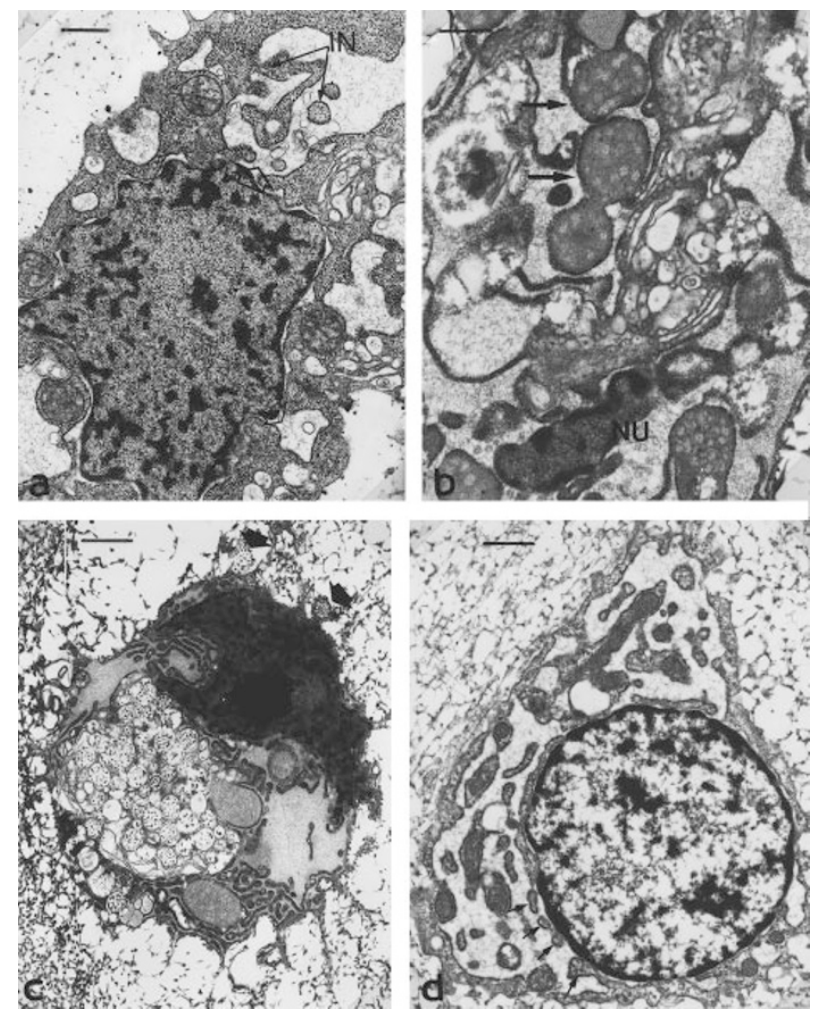

Figure 3 (a) Invaginations of the ground cytoplasm making 'inverted' channels (IN) in the cisterna of RER formed by distension of the outer nuclear membrane (broad blank arrows) in the early dark hypertrophic chondrocyte of 7-d. embryo. Bar: $0.8 \mu \mathrm{m}$. (b) Energetically active mitochondria with fenestrated membranes (arrows) in close vicinity of the Golgi complex in the dark chondrocyte of 14-d. embryo. NU - nucleus. Bar: $0.5 \mu \mathrm{m}$. (c) A hypertrophic chondrocyte containing a prominent Golgi complex embracing a huge sack of secretion vesicles in co-operation with large dark mitochondria; some secretion vesicles are excavating into the cell exterior (arrows). 16-d. embryo. Bar: $0.7 \mu \mathrm{m}$. (d) A very early dark chondrocyte in the growth plate of 7 d. embryo. Segmentation of nuclear envelope (arrows), typical cytoplasmic profile. Bar: $1 \mu \mathrm{m}$
Undeveloped accessorial nucleoli were regularly found in dark cells (Figure 2c).

Cell membranes Light hypertrophic chondrocytes sometimes intensively budded the outer nuclear envelope, providing new cisternae of RER (Figure 2d). In dark chondrocytes, in addition to this process, multiple invaginations of the ground cytoplasm into the dilatated cisternae, formed by distending the outer nuclear envelope, took place (Figure 3a). These invaginations, stud with ribosomes, formed the 'inverted channels' (with ribosomes attached to the inner surface) giving the cytoplasm of dark cells a typical character (Figures 1c, 2b, 3a,d, 4a,c). Defects in the nuclear membrane were usual in dark cells. Its segments rolled into small inverted buds (arrows in Figure 3d) and/or dissoluted together with adjacent chromatin clumps releasing pore complexes (Figure

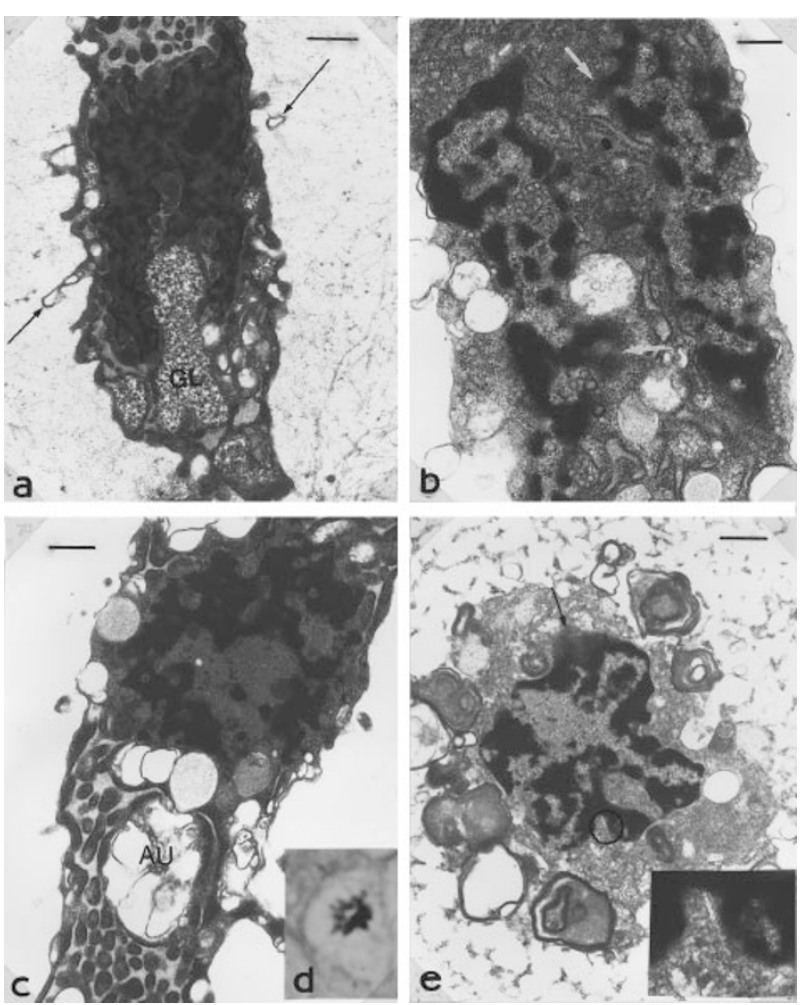

Figure 4 (a) Sandwich-like amitotic segregation of nucleus in a dark chondrocyte in the proliferating zone of 14-d. embryo. GI - glycogen store; arrows - plasmelemmal blebs. Bar: $0.7 \mu \mathrm{m}$. (b) Sandwich-like bi-nuclearity in a dark cell with signs of degeneration: local dissolution of the chromatin and adjacent nuclear membrane (arrows), vacuolation of mitochondria. 15-d. embryo. Bar: $0.35 \mu \mathrm{m}$. (c) A pro-metaphase-like arrangement of chromosomes, enclosed in nuclear envelope. Note the absence of a nucleolus; the dense degenerative karyoplasm, the typical dark cell appearance of the RER, autophagosome $(A U)$, swollen mitochondria. Bar: $1 \mu \mathrm{m}$. (d) A typical view of the metaphase-like arrangement of the very condensed chromatin within matrix lacuna on a cell imprint (DNA plus proteoglycan staining), magnification $\times 2000$. Both (c,d) samples from a 14-d. chick embryo. Bar: $0.6 \mu \mathrm{m}$. (e) A 'clover leaf' (metaphase-like) arrangement of the very compacted chromatin within a degenerative chondrocyte. Encircled-a spindle element attached to the chromatin (enlarged on insert, magnification $\times 30000$ ). Note that the nuclear envelope is generally preserved, however, it is locally lysed together with the adjacent chromatin (arrow). Bar: $0.7 \mu \mathrm{m}$ 
4b). Small blebbings (zeiosis) of the cell membrane was another usual activity of dark cells (Figures 2b, 4a); more seldom the cytoplasm was seen just disintegrating.

Mitochondria, glycogen The light chondrocytes possessed small mitochondria and relatively scarse rosettes of glycogen. In dark cells, the characteristic features were: enrichment with glycogen (Figure 1c) and presence of large, activated mitochondria. These had a dark matrix and well developed cristae (Figures 2b, 3c, 6b), sometimes fenestrated (Figure $3 b)$, suggesting high energetic activity. However, in advanced dark cells, the swollen mitochondria (Figures 1b, 4b, c) and excessive storing of glycogen (Figure $4 a$ ) signalled degeneration.

Golgi complex This was often the prominent organelle of dark cells (Figures $3 a-c$ ). Sometimes all other components, including nucleus, looked like a small adduct to a hypertrophied 'belly' of Golgi and sacks of secretion vesicles. In some places, not only numerous secretion vesicles but the whole cisternae, were seen about to rupture or excavate into the cell exterior.

\section{Transition light-dark cells}

Cells with a transition morphology from light to dark cells (or vice versa?) were encountered only in early embryos. These cells possessed the two general features of dark cells - the characteristic pattern of the chromatin compaction and the 'inverted' membranes, however their karyoplasm and ground cytoplasm were not condensed (Figure 3d).

\section{Cell divisions}

In the proliferating region of normal embryos the light chondrocytes were actively dividing by mitosis. The dark cells were never encountered in normal mitosis. However, sandwich-like amitotic nuclear segregation was found in dark cells both by light microscopy and TEM (Figure 4a). Further, these nuclei often lost nucleoli and underwent hypercompaction of the chromatin followed by its dissolution (Figure 4b). The dark cells sometimes displayed a chromatin arrangement reminiscent of a highly condensed prometaphase or metaphase (Figures $4 \mathrm{c}-\mathrm{e}$ ). As in normal mitosis, these figures lacked nucleoli, however were fully or partially enclosed by a nuclear envelope. Occasionally, separate microtubules were found attached between lobes of the chromosome-like chromatin clumps (Figure 4e). This pattern, known as a 'clover leaf' has been described in some tumours and irradiated cells (reviewed by Ghadially, 1988). Correspondingly, the metaphase-like chromatin figures in interphasic cells (known in light microscopy also as Rabl orientation) were often found on cell imprints (Figure 4d). The process of the chromatin compaction into these figures had been analyzed previously by image analysis and had been found to resemble closely the assemblance of a true mitotic metaphase (Erenpreisa et al, 1993). All division-like forms of the chromatin arrangement had features of aberrancy: minimal presence or absence of spindle elements, preservation or only partial loss of nuclear membrane, combined with hypercompaction and dissolution of the chromatin and other signs of cell degeneration.

\section{RNA synthesis and DNA content}

RNA synthesis, tested in the proliferating and maturing regions of $15-\mathrm{d}$ chick embryo by $1 \mathrm{~h}$ incorporation of $\left[{ }^{3} \mathrm{H}\right]-$ uridine, indicated that the dark cells were including the nuclear label at a much lower rate than the light cell nuclei. The average silver grain number was $1.29 \pm 0.24(n=85)$ in dark cells and $4.97 \pm 0.75(n=75)$ in light cells.

By DNA content, both light and dark cells in the proliferating regions of 14 to 18-d chick embryos were mostly diploid, however about one quarter-one third of them were tetraploid. DNA histograms of light cells (Figure $5 A$ ) indicated the DNA replicative phase of both di- and tetraploid chondrocytes. By contrast, the S-phase was absent from the DNA histograms of dark cells (Figure 5B) measured in the same areas of the chicken growth plate. The other difference was the presence of a hypodiploid fraction $\left(A_{0}\right)$ indicating loss of DNA in about $8-10 \%$ of dark cells. Often the dark cells appeared in the light microscope as bi-nuclear, or sandwich-like. One third of these nuclei (very narrow and with vivid defects of chromatin) contained strongly hypodiploid, often unequal DNA content. Similarly, rough defects of structure and hypodiploid amount of DNA were found in metaphase-like nuclei of dark cells.
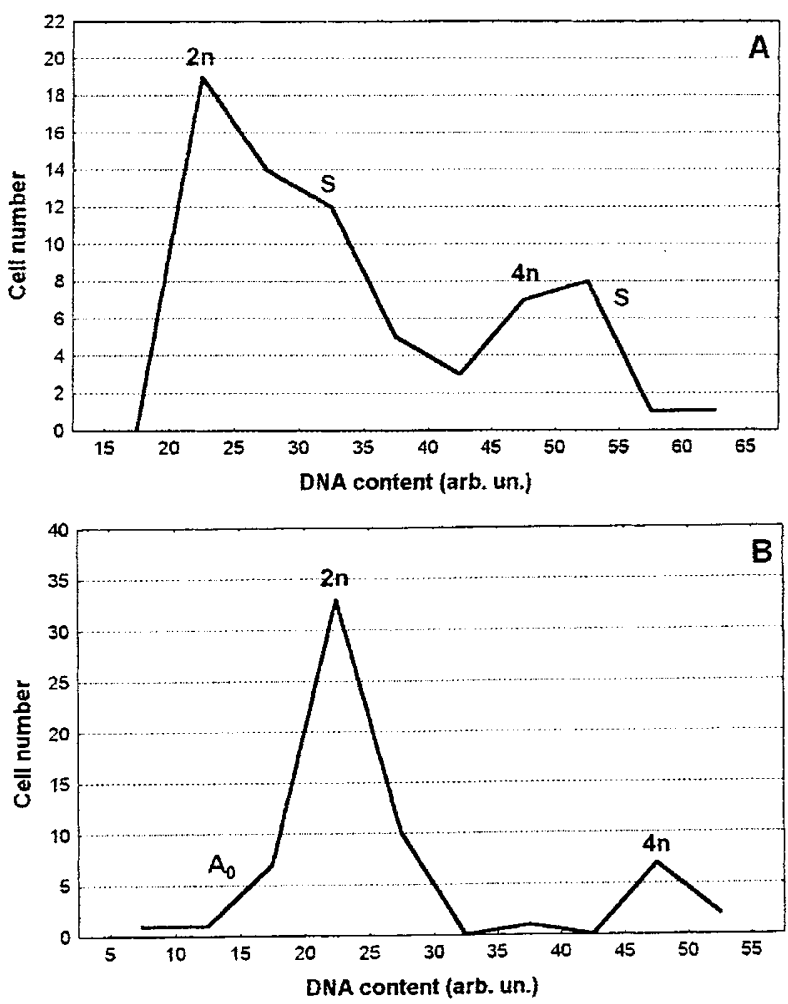

Figure 5 Distribution of DNA contents in the population of light (a) and dark (b) cells of the proliferating zone of two 14-d. chick embryos. $2 n$ corresponds an average value (23.5 arb.units) of a chicken diploid erythrocyte nucleus; $4 \mathrm{n}$ - tetraploid value; Ao - hypodiploid degenerative fraction; $S$ - replicative fraction. Similar distributions were found for 15.- and 18.-d. embryos 


\section{Cell death}

After close EM examination of 11 growth plates of chick embryos, cell after a cell, chondrocytes with the 'classic' apoptotic morphology were not found, although apoptotic cells were present at the marrow/cartilage interface. By contrast, degenerative dark cells of the described morphology were often found. As mentioned above, their compacted chromatin usually underwent dissolution. Correspondingly, karyolytic dark cells with typical cytoplasmic profile were occasionally encountered in the immediate neighbourhood with normal light cells (Figure 6a). Sometimes, the most shrunk dark cells mimicked under low EM resolution an apoptotic morphology, however, their chromatin was not entirely condensed or sharply delineated as in apoptotic cells and they always displayed a great deal of cytoplasmic activity, including autophagy (Figure 1b). Sometimes, such a dark cell, still recognizable by cytoplasmic and nuclear features, was seen engulfed by a light chondrocyte (Figure 6b). Nevertheless, all these cases were different from conventional ('classic') apoptosis of chondrocyte. The latter had been observed, using the same fixation, in hypertrophic chondrocytes in organ culture (Roach et al, 1995; Erenpreisa and Roach, 1996).

By direct test for DNA strand breaks, the larger nuclei of light hypertrophic chondrocytes of normal chick embryo did not contain label, while about two thirds of smaller dense nuclei of dark cells contained a limited label, which was tentatively above a background. In the positive control for DNA strand breaks using the DNAse I mild digestion, the same technique provided a heavy nuclear label (data not shown).

\section{Discussion}

Preservation of dark cells of the described morphology, using any fixative applied, confirmed that the dark cells were not simply a fixation artifact. Their proneness to shrink in chemical fixatives and high vulnerability in organic solvents, as also indicated by other authors (Glaso and Hovig, 1987; Ghadially,

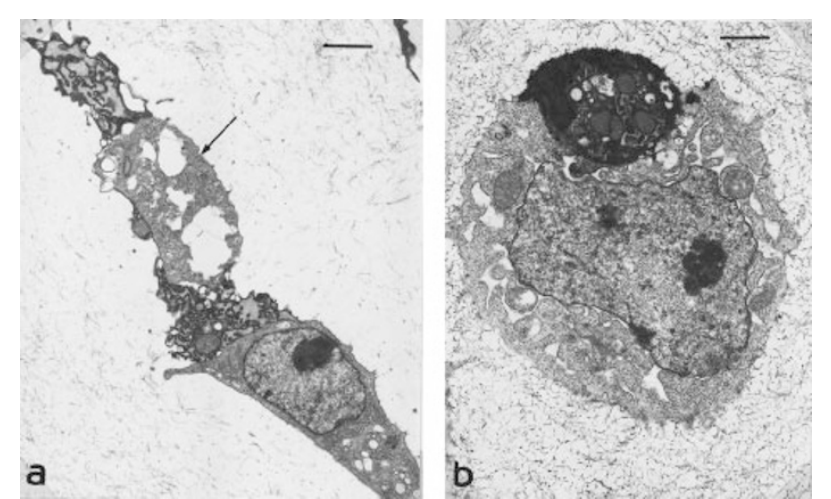

Figure 6 Two different degeneration patterns of dark cells. (a) Karyolytic dark cell (arrow) forming a pair with a conventional chondrocyte in a proliferative zone of 18-d. embryo. Bar: $0.8 \mu \mathrm{m}$. (b) Karyopycnotic dark cell (note the presence of large mitochondria) being engulfed by a light chondrocyte in a resting zone of $9-\mathrm{d}$. embryo. Bar: $1.5 \mu \mathrm{m}$

1988), may be associated with the impaired membranes. Cells of a similar morphology in the mouse rib were considered by Holtrop (1972) to represent the terminal differentiation stage of hypertrophic chondrocytes. However, we have found them in all differentiation layers of a growth plate.

The emergence of dark cells in the growth plate already in the sub-perichondrial layer might suggest that they represent a particular subset of chondrocytes. The strongest evidence against this notion is the finding of both light and dark cells in postmitotic doublets in the proliferation zone, suggesting their origin from one mother cell.

The dark chondrocytes displayed a peculiar set of the seemingly contradictory characteristics - high synthetic and secretory activity of the cytoplasm, energetically supported by active mitochondria and synthesis of glycogen, on the one hand, and signs of inactivation and degeneration, mostly nuclear, on the other. While indented nuclei and scattered clumping of chromatin has been described by some authors in early dark cells of other tissues (e.g. Harmon, 1987), the active profile of cytoplasm, with a few exceptions (Tewari and Bourne, 1982; Ohtsuka and Murakami, 1996), has not been noticed. The same refers to 'inverted' channels of RER. In fact, most authors only described the morphology of late dark cells. However, the data of Chentsov and Kosyh (1995) on high energetic level of mitochondria in dark rat hepatocytes are consistent with our observations in chick chondrocytes. The fact that a high proportion of the dark cells displayed considerable cytoplasmic activity may represent a peculiarity of the chicken growth plate chondrocytes.

Our observations showed that dark chondrocytes had a greater secretory function than the light ones. It is also quite evident from the cytological features and RNA autoradiography, that in the chain: RNA transcription protein synthesis - secretion, the balance in dark chondrocytes was shifted to the last phase of the functional life cycle. It is possible that this corresponded to intensive translation of a few long-lived specific mRNAs provided by the rich ribosomal supply.

At the same time, the absence of normal cell divisions, low RNA synthesis, serrated nuclear envelope and chromatin margination, nucleolar segregation or compaction, impaired membranes, blistering plasmalemma, autophagy and often swollen mitochondria, are all indicative of degeneration.

Most investigators suggested that the most advanced dark cells underwent some kind of shrinkage necrosis (Kerr et al, 1987). Our observations, including low level of DNA strand breaks, which is consistent with quiescence or necrosis rather than apoptosis (Gorzyca et al, 1993), seem to fit this view. However, this was not quite a typical necrosis, since the cytoplasm was not swollen. On the contrary, the dense karyoplasm and hyaloplasm, enrichment with free ribosomes, and phagocytosis by neighbours are the features shared with apoptotic degeneration. So, the dark cells displayed a mixed type of cell death, which could shift in some cases to a predominantly karyolytic type (seen on Figure 6a) and in other cases, to a karyopycnotic type (seen on Figure 6b). 
The peculiarity of the chromatin arrangement in dark chondrocytes should be considered. Our present studies of the rabbit growth plates are showing the same chromatin patterns (unpublished). This similarity indicates that this type of nuclear compaction is not due to the primary structure of the avian genome but has a more general nature. Flocculation of diffuse chromatin has been described as characteristic of the early stress preceding both apoptotic and necrotic damage, as well as the induction of differentiation in various cells (Wyllie, 1988; Erenpreisa, 1991). The chromomeric-chromonemic condensation of the chromatin may be interpreted as being associated with the early prophasic arrest or premature partial condensation of chromosomes. More than that, our previous and present observations showed that in many cases, the next change of the dark cell nucleus, associated with loss of nucleoli and partial dissolution of nuclear envelope was either amitotic segregation and/or the metaphase-like arrangement of the chromatin. It should be noted, however, that, as well as the majority of dark cells, these 'metaphases' ('clover leafs', 'Rabl orientations') were diploid (or hypodiploid) by DNA content. This suggests that the dark cells were undergoing some kind of premature mitosis associated with degeneration.

Premature activation of the cell cycle protein kinase, found in some cases of apoptosis (Shi et al, 1994), has led to the view of apoptosis as an abortive mitosis (reviewed by Evan et al, 1995). Deregulation of the cell cycle components has been shown to cause an aberrant form of mitosis associated with death and called 'mitotic catastrophe' (reviewed by King and Cidlowski, 1995). Kondo (1995) suggested a mitotic catastrophe model for cells dying in G1/G2 phase by short circuiting to the $M$ phase. This model is likely supported by our observations. Also Dini et al (1996) described cellular degeneration, resulting from apoptogenic treatments of tumour cells, which appeared to be midway between mitotically condensed chromosomes and apoptotically condensed chromatin. Here we have similar findings in normal development. Therefore, the type of the cell death described above is likely to be programmed. It seems to provide a mechanism for the dissociation of nuclear and cytoplasmic activities allowing the final burst of secretion.

In conclusion, the answers to the questions asked when starting the investigation of dark chondrocytes are as follows: (1) They are not generated by fixation but are prone to fixation shrinkage. (2) Dark cells are not a particular subset of chondrocytes. (3) They are not the terminal stage in the vertical differentiation of chondrocytes through the growth plate. (4) Rather, dark chondrocytes seem to represent a terminal state in a horizontal differentiation, that is in a functional cycle of a chondrocyte, which is exhibited as a very intense and self-sacrificing secretion.

\section{Materials and Methods}

The proximal femoral growth plates were studied in 11 chick embryos (7d.-2; 9-d.-1; 11-d.-1, 14-d.-2; 15, 16, 17, 18 and 19-d. - one sample for each day). Fixation of the highly hydrated embryonic cartilage tissue, and hypertrophic chondrocytes in particular, posed considerable difficulties because the cells collapsed, unless cationic compounds precipitating proteoglycans were added (Hunziker et al, 1983). Hence samples were fixed for TEM in $2.5 \%$ glutaraldehyde (GA) in $0.05-0.1 \mathrm{M}$ cacodylate buffer containing either $0.7 \%$ Ruthenium hexamethylene trichloride (GA/ $\mathrm{RHT}$ ), as recommended by Hunziker et al (1983), or $1 \%$ Alcian blue (GA/ AB) (details in Erenpreisa and Roach, 1996). Samples were post-fixed with uranyl and osmium and embedded in Spurr resin. In separate experiments, $6.8 \%$ sucrose or $2 \mathrm{mM} \mathrm{CaCl} 2$ were added to the first fixative. The material was oriented while embedding; circular and longitudinal semi-thin and thin sections were obtained, stained with Toluidine blue (TB) or lead citrate, respectively.

Cell imprints of growth plate chondrocytes were taken for morphological and DNA cytometric examination by gently pressing longitudinal slices upon defatted microscopic slides, fixing in ethanol/ acetone $(1: 1)$ for $30 \mathrm{~min}$ and air-drying. Morphological staining was performed with $0.05 \%$ TB (Gurr) in 50\% citrate-phosphate buffer pH 5.0 after treatment with $0.1 \mathrm{~N} \mathrm{HCl}, 4^{\circ} \mathrm{C}, 10 \mathrm{~min}$. Quantitative staining of DNA with TB in the same buffer $\mathrm{pH} 4.0$ was done after pretreatment with $5 \mathrm{~N} \mathrm{HCl}, 21^{\circ} \mathrm{C}, 30 \mathrm{~min}$ followed by 2,4-dinitrophenyl-hydrazine treatment (saturated in $1 \mathrm{~N} \mathrm{HCl}, 0^{\circ} \mathrm{C}, 10 \mathrm{~h}$ ). In addition to DNA staining, the method stained proteoglycans weakly, so that the chondrocyte nuclei could be recognized in clear lacunae within the ECM matrix (for details, Erenpreisa et al, 1991). The influence of fixation with $\mathrm{GA} / \mathrm{RHT}, \mathrm{GA} / \mathrm{AB}$, ethanol/acetone, methanol, and of acid treatment on the morphology of dark cells in imprints was studied. For RNA autoradiography, 15-d. chick embryo femur slices were incubated at $37^{\circ} \mathrm{C}$ in a culture medium with $5 \mu \mathrm{Ci} / \mathrm{ml}$ of $\left[{ }^{3} \mathrm{H}\right]$ uridine for $1 \mathrm{~h}$, fixed and embedded in Spurr resin. Semithin sections, lightly pre-stained with TB, were exposed with K-5 llford emulsion for 5 weeks. The number of RNAassociated silver grains per nucleus was counted under oil immersion in light and dark chondrocytes of the proliferating and maturing zones.

To demonstrate DNA breaks, the cell imprints were fixed in methanol/acetone at $-20^{\circ} \mathrm{C}$ for $10 \mathrm{~min}$ and DNA strand-breaks were end-labelled by the TUNEL method as described by Gavrieli et al (1992), except that the Boehringer Mannheim digoxigenin-DNA labelling kit, was used and proteinase pre-treatment was omitted.

DNA cytometry was performed with a 2-wave-length ultraviolet cytophotometer (MCFU-1, LOMO), using immersion magnifications and corresponding nuclear plugs, on two samples of 14-d., two samples of 15-d., and 1 sample of 18.-d. chicken material. The optical densities $(\lambda=570 \mathrm{~nm})$ of the cell light and dark nuclei, in clearly visible lacunae were measured in the proliferating and maturing zones of the growth plates.

\section{Acknowledgements}

The authors gratefully acknowledge the support of the Royal Society (London) for scientific visits between Southampton and Riga. We also appreciated the discussion and advice of Prof Robin Stockwell (retired) and Dr Boris Zhivotovsky (Karolinska Institute, Stockholm). The excellent technical assistance of Mrs Janice Baker, Miss Sue Cox, Mr Nick Barnett (University of Southampton) and Mr Vladislavs Zelchs (Latvian University) is greatly acknowledged.

\section{References}

Aizawa T, Tanaka Y and Kokubun S (1997) Apoptosis and proliferation of growth plate chondrocytes in rabbits. J. Bone Joint Surg. 79B: 483-486

Bronkers ALJJ, Goei W, Luo G, etal (1996) DNA fragmentation during bone formation in neonatal rodents assessed by transferase-mediated end labelling. J. Bone Min. Res. 11: 1281-1291 
Dark chondrocytes

$\mathrm{J}$ Erenpreisa and HI Roach
Cancedda R, Descalzi-Cancedda F and Castagnola P (1995) Chondrocyte differentiation. Int. Rev. Cytol. 159: 265-358

Chentsov YS and Kosyh MI (1995) Bioenergetic, cytochemical, and tinctorial features of normal hepatocytes and after functional load, adaptation to environment change, and nonspecific damage. Tsytologia (Cytology) 37: $1123-1135$ (russ.)

Cooper EH, Bedford AJ and Kenny TE (1975) Cell death in normal and malignant tissues. Adv. Canc. Res. 21:59-120

Dini L, Coppola S, Ruzutti MT and Ghibelli L (1996) Multiple pathways for apoptotic nuclear fragmentation. Exp. Cell Res. 223: 340-347

Evan GI, Whyte Mand Harrington E (1995) Apoptosis and the cell cycle. Curr. Opinion Cell Biol. 7: 825-834

Erenpreisa Je (1989) Two mechanisms of chromatin compaction. Acta histochem. 86: $129-135$

Erenpreisa Je, Zhukotsky A, Butusova N et al (1991) Accumulation of DNA within chromocentres of terminally differentiating chick embryo chondrocytes. Acta histochem. 90: 113-119

Erenpreisa Je and Zhukotsky A (1993) Interphase genome as the active space: Chromatin dynamics during chick embryo chondrogenesis. Mech. Ag. \& Dev. 67 : $21-32$

Erenpreisa Je, Zhukotsky A and Kozlov A (1993) The chromatin network: Image analysis of differentiating chick embryo chondrocytes. Eur. J. Histochem. 37: $139-147$

Erenpreisa Je and Roach HI (1996) Epigenetic selection as a possible component of transdifferentiation. Further study of the commitment of hypertrophic chondrocytes to become osteogenic cells. Mech. Ag. \& Dev. 87: 165-182

Farnum C, Turgai J and Wilsman N (1990) Visualization of living terminal hypertrophic chondrocytes of growth plate cartilage in situ by differential interference contrast microscopy and time-lapse cinematography. J. Orthop. Res. 8: 750-763

Ganote CE and Moses HL (1968) Light and dark cells as artifacts of liver fixation. Lab. Inves. 18: $740-745$

Gavrieli Y, Sherman Y and Ben-Sasson SA (1993) Identification of programmed cell death in situ via specific labeling of nuclear DNA fragmentation. J. Cell Biol. 119: 493-501

Ghadially FN (1988) The dark-light cell phenomenon. In: Ultrastructural pathology of the cell and matrix (Butterworths), 3rd. ed., Vol. 2, pp. 954-961

Gibson GJ, KohlerWJ and Schaffler MB (1995) Chondrocyte apoptosis in enchondral ossification of chick sterna. Dev. Dynamics 203: 468-476

Glaso M and Hovig T (1987) A light and electron microscopical study with special reference to 'dark cells' and epidermal carcinogenesis. Virchow's Arch. B 54: $73-88$
Gorzyca W, Gong J and Darzynkiewicz Z (1993) Detection of DNA strand breaks in individual apoptotic cells by the in situ terminal deoxynucleotidyl transferase and nick translation assays. Canc. Res. 53: 1945-1951

Hadjiolov AA (1985) Nucleolus and ribosome biogenesis: Cell biology monographs. (Wien, N.-Y.: Springer Verl.)

Harmon BV (1987) An ultrastructural study of spontaneous cell death in a mouse mastocytoma with particular reference to dark cells. J. Pathol 158: 345-355

Hatori M, Klatte KJ, Teixeira CC and Shapiro IM (1995) End labeling studies of fragmented DNA in the avian growth plate: Evidence of apoptosis in terminally differentiated chondrocytes. J. Bone Min. Res. 10: 1960-1968

Holtrop ME (1972) Ultrastructure of the epiphyseal plate. II. The hypertrophic chondrocyte. Calc. Tiss. Res. 9: 140-151

Hunziker EB, Herrmann W and Schenk R (1983) Ruthenium hexamine trichloride (RHT)-mediated interaction between plasmalemmal components and pericellular matrix proteoglycans is responsible for the preservation of chondrocytic plasma membranes in situ during cartilage fixation. J. Histochem. Cytochem. 31 $717-727$

Johannisson $E$ (1968) The foetal adrenal cortex in the human. Its ultrastructure at different stages of development and in different functional states. Acta Endocrinol. (Suppl.130) [Copenh] 58: 7-107

Kerr JFR, Searle J, Harmon BV and Bishop CJ (1987) Apoptosis. In: Perspectives on mammalian cell death. C.S. Potten ed. (Oxford: Univ. Press), pp.93-128

King KL and Cidlowski JA (1995) Cell cycle and apoptosis - common pathway to life and death. J. Cell. Biochem. 58: 175-180

Kondo S (1995) Apoptosis by antitumour agents and other factors in relation to cellcycle checkpoints. J. Radiat. Res. 36: 56-62

Otsuka A and Murakami T (1996) Dark neurons in the mouse brain: an investigation into the possible significance of their variable appearance within a day and their relation to negatively charged cell coats. Arch. Histol. Cytol. 59: 79-85

Parsons DF, Marko MM, Braun SJ et al (1983) 'Dark cells' in normal, hyperplasic, and promoter-treated mouse epidermis studied by conventional and high voltage electron microscopy. J. Invest. Dermatol. 81: 62-67

Roach HI, Erenpreisa Je and Aigner T (1995) Osteogenic differentiation of hypertrophic chondrocytes involves asymmetric cell divisions and apoptosis. J. Cell Biol. 131: 483-494

Shi L, Nishioka WK, Thng J et al (1994) Premature p34 $\mathrm{cdc}^{2}$ activation required for apoptosis. Science 263: 1143-1145

Tewari HB and Bourne GH (1983) Histochemical studies of the 'dark' and 'light' cells of the cerebellum of rat. Acta Neuropathol.(Berl.) 3:1-15

Wyllie AH (1988) Apoptosis. ISI Atlas Science. Immunol. 1: 192-196 